Arq. Bras. Med. Vet. Zootec., v.63, n.1, p.165-170, 2011

\title{
Comportamento alimentar de cabras alimentadas com diferentes teores de fibra em detergente neutro
}

[Feeding behavior of goats fed with different NDF levels]

\author{
V.B. Silva ${ }^{1}$, C.E.M. Fonseca ${ }^{2}$, M.I.V. Almeida ${ }^{3}$ L. S.G. Brasileiro ${ }^{4}$, F.N. Godoi ${ }^{2}$, M.S. Mendonça ${ }^{5}$ \\ ${ }^{1}$ Aluna de pós-graduação - EV-UFMG - Belo Horizonte, MG \\ ${ }^{2}$ Universidade Federal Rural do Rio de Janeiro - Seropédica, RJ \\ ${ }^{3}$ Universidade Federal do Espírito Santo - Vitória, ES \\ ${ }^{4}$ Aluno de graduação - UFRRJ - Seropédica, RJ \\ ${ }^{5}$ Zootecnista autônoma
}

\begin{abstract}
RESUMO
Avaliou-se o comportamento alimentar de cabras alimentadas com dietas que continham diferentes teores de fibra em detergente neutro (FDN) - 49, 54, 59, 64 e 69\% -, em função de diferentes teores de inclusão de resíduo úmido de cervejaria (RUC), em substituição ao concentrado da dieta - 0, 25, 50, 75 e $100 \%$. Foram utilizadas cinco cabras mestiças Boer x Saanen e cinco cabras Saanen distribuídas em dois quadrados latinos 5x5. O volumoso utilizado foi feno de tifton, com relação volumoso: concentrado de 40:60. As observações foram feitas em períodos de 20 minutos até o tempo total de 24 horas. Os animais dos diferentes genótipos não apresentaram diferenças no comportamento alimentar. Os tempos gastos com alimentação e com ociosidade não diferiram quanto aos teores de FDN na dieta, no entanto resposta linear crescente foi observada para os tempos despendidos com ruminação e com a mastigação total com o aumento dos teores de FDN e RUC na dieta. As dietas com os teores de 49 e 64\% apresentaram menor tempo de ruminação quando comparadas com a dieta com teor de $69 \%$ de FDN, com resultados respectivos de 218, 268 e 366 minutos de ruminação. Isso contribuiu para diminuir a eficiência (min/kg MS e FDN) de alimentação, ruminação e mastigação. Conclui-se que o resíduo úmido de cervejaria pode ser usado como fonte de fibra efetiva na dieta de cabras.
\end{abstract}

Palavras-chave: caprino, fibra efetiva, tempo de ruminação, resíduo úmido de cervejaria

\begin{abstract}
The feeding behavior was evaluated in lactating goats fed diets with different levels of NDF (neutral detergent fiber) 49, 54, 59, 64, and 69\%, in function of different levels of WBG (wet brewery grains) 0 , 25, 50, 75, and 100\%. Five goats Boer $x$ Saanen and five Saanen goats were randomly distributed in two $5 \times 5$ latin squares. The used roughage tifton hay in a roughage:concentrate rate of 60:40. The feeding times, idle time, and rumination time were evaluated each 20 minutes during 24 hours. The feeding behavior was not influenced by genotypes. The feeding times and idles times were not affected by increasing NDF levels, however, the rumination time and total chewing time had growing linear effect with increase of NDF levels in which the levels 49 and 64\% showed shorter rumination time than 69\% NDF, 218, 268, and 366min respectively. It decreased the feeding, rumination and chewing efficiency (min/ $\mathrm{kg} \mathrm{DM}$ and NDF). It was concluded that WBG can be used as a source of effective fiber in diets of goats.
\end{abstract}

Keywords: goat, effective fiber, rumination time, wet brewery grains

Recebido em 11 de julho de 2010

Aceito em 10 de fevereiro de 2011

E-mail: basoniveri@yahoo.com.br

Apoio: FAPERJ 


\section{INTRODUÇÃO}

O caprinocultor tem utilizado resíduos agroindustriais na nutrição do rebanho. Tendo em vista que a nutrição constitui a base para o sucesso do sistema de produção, devem-se adotar estratégias que visem aumentar a eficiência de utilização dos nutrientes, aliadas à redução dos custos com alimentação.

Dentre os resíduos agroindustriais utilizados, destaca-se o resíduo úmido de cervejaria (Brochier e Carvalho, 2009), que se caracteriza como um resíduo fibroso, com 59 a 65\% de fibra em detergente neutro (FDN) (West e Martin, 1994; Cabral Filho, 1999; Geron et al., 2008). No entanto, o resíduo úmido de cervejaria (RUC) apresenta tamanho de partículas diminutas, inerentes ao processamento industrial. Animais que ingerem volumosos com tamanho de partículas reduzidas apresentam menor tempo de ruminação, devido à baixa efetividade da fibra.

Para avaliação do tempo de ruminação, é pertinente estudar o comportamento ingestivo, que pode ser feito por meio de observações do tempo de alimentação, ruminação e ociosidade do animal. Existem variadas técnicas de registros de dados, como observações visuais, registros semiautomáticos e automáticos com utilização de câmeras filmadoras. Alguns trabalhos de observação do comportamento alimentar utilizaram diferentes escalas de tempo: de cinco minutos (Bürger et al., 2000), sete minutos (Deswysen et al., 1993), 10 minutos (Costa et al., 2003) e 15 minutos (Fischer et al., 1998). Carvalho et al. (2004), objetivando definir o intervalo de observação ideal no comportamento alimentar de cabras, não encontraram diferença nos tempos de alimentação, ruminação e ociosidade para intervalos de observações de cinco, 10, 15 e 20 minutos.

O objetivo deste trabalho foi avaliar o comportamento alimentar de cabras de genótipos distintos, alimentadas com diferentes teores de FDN, em função de diferentes teores de RUC da dieta.

\section{MATERIAL E MÉTODOS}

O experimento foi realizado no setor de caprinocultura do Instituto de Zootecnia da Universidade Federal Rural do Rio de Janeiro RJ. Foram utilizadas 10 cabras em final de lactação, sendo cinco da raça Saanen (S), com média de peso de $53,0 \pm 9,7 \mathrm{~kg}$, e cinco mestiças Boer $\mathrm{x}$ Saanen (BS), com média de peso de $51,0 \pm 6,2 \mathrm{~kg}$, distribuídas em dois quadrados latinos $5 \times 5$. As cabras foram confinadas em gaiolas metabólicas individuais providas de comedouro e bebedouro e tratadas inicialmente contra ecto e endoparasitos.

Os tratamentos foram distribuídos em um esquema fatorial $2 \times 5$, dois genótipos e cinco teores de FDN - 49, 54, 59, 64 e 69\%. O teor de $49 \%$ referiu-se à fonte forrageira (feno de tifton), e os teores acima deste foram acrescidos por fonte não forrageira, oriundos do RUC em substituição ao concentrado da dieta.

A dieta foi oferecida com o intuito de fornecer a relação volumoso:concentrado de 60:40, no entanto, no decorrer do experimento, essa relação foi invertida, ou seja, o consumo real foi de 40:60, devido ao oferecimento do volumoso separado do concentrado, aliado à seletividade dos animais pelo concentrado e pelo RUC. O concentrado foi formulado visando prepará-lo com o mesmo teor de proteína do RUC, para que as dietas oferecidas fossem isoproteicas (Tab. 1).

Cada período experimental teve duração de 15 dias, sendo 10 dias de adaptação à dieta e cinco dias de coleta de dados, em que foi observado o consumo de alimentos. No $13^{\circ}$ dia de cada período experimental, foram realizadas as observações de comportamento alimentar.

Os animais foram alimentados às nove e às 15 horas, e o consumo foi ajustado para permitir cerca de $20 \%$ de sobras.

Na Tab. 1, encontra-se a composição bromatológica das dietas, de acordo com os níveis de substituição do concentrado pelo RUC. Amostras dos alimentos fornecidos e das sobras foram secas a $65^{\circ} \mathrm{C}$ em estufa de ventilação forçada, durante 72 horas, moídas em moinho tipo Willey, com peneiras de $1 \mathrm{~mm}$ e acondicionadas em sacos plásticos devidamente identificados. Foram realizadas análises de matéria seca (MS), matéria orgânica (MO), proteína bruta $(\mathrm{PB})$ e extrato etéreo (EE), segundo recomendações da AOAC (Official..., 1984), e as análises de FDN, conforme Van Soest et al. (1991). 
Tabela 1. Composição bromatológica dos alimentos das dietas, de acordo com os teores de resíduo úmido de cervejaria (RUC) em substituição ao concentrado

\begin{tabular}{|c|c|c|c|c|c|}
\hline \multirow{2}{*}{ Item } & \multicolumn{5}{|c|}{ Alimentos } \\
\hline & \multicolumn{2}{|l|}{ RUC } & \multicolumn{2}{|l|}{ Concentrado $^{1}$} & Feno \\
\hline FDN & \multicolumn{2}{|l|}{58,0} & 16,0 & & 76,0 \\
\hline MS & \multicolumn{2}{|l|}{22,0} & 88,0 & & 80,0 \\
\hline MO & \multicolumn{2}{|l|}{95,9} & 96,4 & & 94,9 \\
\hline $\mathrm{PB}$ & \multicolumn{2}{|l|}{20,3} & 20,1 & & 5,1 \\
\hline \multirow[t]{3}{*}{$\mathrm{EE}$} & 9,9 & & 3,1 & & 1,2 \\
\hline & \multicolumn{5}{|c|}{ Dietas de acordo com teores de RUC (\%) } \\
\hline & 0 & 25 & 50 & 75 & 100 \\
\hline FDN & 49,00 & 54,00 & 59,00 & 64,00 & 69,00 \\
\hline MS & 83,20 & 76,60 & 70,00 & 63,40 & 56,80 \\
\hline MO & 95,50 & 95,40 & 95,40 & 95,50 & 95,50 \\
\hline $\mathrm{PB}$ & 11,10 & 11,10 & 11,10 & 11,10 & 11,20 \\
\hline $\mathrm{EE}$ & 1,90 & 2,60 & 3,30 & 4,00 & 4,70 \\
\hline $\mathrm{CT}$ & 81,20 & 80,60 & 80,00 & 79,40 & 78,80 \\
\hline
\end{tabular}

${ }^{1}=54 \%$ de fubá de milho, $31 \%$ de farelo de soja, $14 \%$ de farelo de trigo e $1 \%$ de mistura mineral

As variáveis observadas foram: tempo gasto em minutos com alimentação, ruminação e ociosidade, obtidos por observações dos animais a cada 20 minutos, até completar um total de 24 horas, conforme proposto por Carvalho et al. (2004). Na observação noturna, o ambiente foi mantido com iluminação artificial superficial, procurando não interferir no comportamento individual das cabras.

O tempo de mastigação total foi calculado somando-se o tempo de alimentação ao tempo de ruminação. As eficiências de alimentação, ruminação e mastigação foram calculadas em função do tempo gasto em minutos para consumo em kg de MS e FDN. Os dados de observação alimentar foram submetidos às análises de variância e de regressão, utilizandose SAEG (Sistema..., 1999) e SISVAR (Ferreira, 2000). Para testar a significância dos coeficientes de regressão, foi utilizado o teste t a $5 \%$ de probabilidade.

\section{RESULTADOS E DISCUSSÃO}

Não houve interação de tratamentos versus grupo genético em nenhuma variável analisada. $\mathrm{Na}$ Tab. 2, é apresentado o tempo gasto, em minutos, pelos animais para alimentação, ociosidade, ruminação e mastigação total, em função do teor de FDN da dieta e do genótipo. Não houve diferença entre genótipos $(\mathrm{P}>0,05)$ em nenhuma variável estudada, e o mesmo resultado foi encontrado entre os teores de FDN testados, que também não apresentaram diferença $(\mathrm{P}>0,05)$ no tempo gasto com alimentação e ociosidade, quando comparados à dieta-controle. Esse fato pode ser atribuído ao aumento da porção fibrosa da dieta ocorrido em função do aumento da quantidade de RUC, que apresentou tamanho diminuto das partículas e aumentou a densidade do alimento. Apesar disso, o tamanho do bocado para a ingestão de concentrado e de RUC não variou. Ribeiro et al. (2006) avaliaram o comportamento alimentar de cabritos inteiros consumindo dietas com feno triturado a $4 \mathrm{~mm}$, com $60 \%$ de concentrado na sua composição e não observaram diferenças no tempo gasto com alimentação de animais submetidos ou não à restrição alimentar. Carvalho et al. (2006) encontraram aumento no tempo gasto com alimentação de cabras Alpinas à medida que se aumentou a FDN forrageira da dieta, o que corrobora com a explicação acima, já que o feno oferecido aos animais possuía fibra longa.

O tempo gasto com ruminação foi influenciado pelo aumento do teor de fibra na dieta, aumentando gradativamente o tempo gasto com essa resposta de acordo com o aumento do nível de FDN e de RUC na dieta.

Apesar de o tempo gasto com a ruminação ter apresentado comportamento linear, os dados referentes ao tempo de mastigação não se adequaram a nenhuma curva de regressão, conforme observado na Tab. 2. 
Tabela 2. Tempo médio (min) gasto com alimentação, ócio, ruminação e mastigação total, seus respectivos coeficientes de variação (CV) e equação de regressão ajustada, em função dos diferentes teores de fibra em detergente neutro (FDN) da dieta

\begin{tabular}{lllllllll} 
& \multicolumn{4}{c}{ Nível de FDN } & \multicolumn{5}{c}{ Genótipo } \\
\cline { 2 - 9 } & 49 & 54 & 59 & 64 & 69 & S & BS & CV \\
\hline Alimentação & 394 & 452 & 388 & 388 & 410 & 397 & 416 & 18,12 \\
Ócio & 824 & 716 & 742 & 734 & 660 & 740 & 730 & 15,91 \\
Ruminação $^{1}$ & 218 & 268 & 306 & 314 & 366 & 299 & 289 & 27,40 \\
Mastigação total $^{612}$ & 720 & 694 & 702 & 776 & 696 & 705 & 16,70
\end{tabular}

$\mathrm{S}=$ Saanen; BS= 1/2 Boer $\mathrm{x}$ Saanen; ${ }^{1} \hat{\mathrm{y}}=-109,16+6,84 \mathrm{X}\left(\mathrm{r}^{2}=0,95\right)$, em que $\hat{\mathrm{y}}=$ tempo gasto com cada atividade alimentar e $\mathrm{X}=$ teores de FDN.

O comportamento linear do tempo de ruminação pode ser explicado pelo enchimento físico do rúmen, apesar de o RUC apresentar partículas diminutas, que não influenciaram na quantidade apreendida no bocado, o que refletiu no tempo de alimentação. Essas foram suficientes para causarem efeito sobre o enchimento físico do rúmen, estimulando a ruminação.

Segundo Mertens (1997), o RUC apresenta uma efetividade física da fibra de 8,3\%, o dobro, quando comparado ao milho moído, e de 4,3\%, quando fornecido a vacas leiteiras. Segundo Dado e Allen (1995), o número de períodos de ruminação aumenta com o aumento da quantidade de fibra na dieta, sugerindo haver necessidade de um melhor processamento da digesta ruminal, para maximizar a eficiência digestiva.

Os animais alimentados com a dieta sem inclusão de RUC, contendo 49\% de FDN, gastaram 218 minutos com a ruminação e 612 minutos com a mastigação total. Esses tempos foram inferiores aos citados por Carvalho et al. (2006), que observaram que cabras da raça Alpina, alimentadas com dietas contendo cerca de $48 \%$ de FDN, despenderam 470 minutos com ruminação e 819 minutos com mastigação total. Essa diferença no tempo de ruminação provavelmente ocorreu devido à relação concentrado:volumoso das dietas utilizadas, pois Carvalho et al. (2006) utilizaram dietas com relação entre concentrado e volumoso de 30:70, e no presente trabalho essa relação foi de 60:40.

Na Tab. 3, estão apresentados os consumos médios diários de MS e FDN em g/d e as eficiências de alimentação, ruminação e mastigação, em min/kg MS e min/kg FDN, em função dos teores de FDN da dieta, bem como os seus coeficientes de variação, equações de regressão e seus respectivos coeficientes de determinação.

Foi observado comportamento quadrático para os consumos de MS e FDN (Tab. 3), sendo o consumo máximo para a dieta com 54\% de FDN; acima desse nível, houve redução linear com o aumento de RUC e FDN na dieta. O consumo de FDN também apresentou comportamento quadrático. Estes resultados podem ser explicados por duas teorias.

A primeira é a evidência de que o aumento dos teores de FDN na dieta atua como limitante físico; segundo Mertens (1997), o consumo de alimentos pelos ruminantes depende de fatores físicos que atuam na distensão do aparelho digestivo. Com o enchimento do rúmen e retículo, há o desencadeamento da sensação de saciedade, mediada por mecanorreceptores; por outro lado, a taxa de passagem de alimentos fibrosos tende a ser mais lenta, o que poderia limitar seu consumo, apesar de o aumento de FDN estar vinculado à inclusão de RUC, que apresenta tamanho de partícula diminuta; sua taxa de passagem pode ser maior que a do feno, mas não maior que a do concentrado.

A segunda teoria pode estar ligada ao fato de 0 RUC apresentar alta porcentagem de umidade em sua composição, 78\% (Tab. 1). A ingestão de matéria seca tem relação negativa com a elevação no teor de umidade da dieta (Nutrient..., 2001). A redução do teor de MS na dieta com alta quantidade de RUC pode ser um dos fatores limitantes da utilização do resíduo na formulação das dietas.

A eficiência de ruminação expressa em $\mathrm{min} / \mathrm{kg}$ MS e min/kg FDN foi influenciada pelos teores de FDN da dieta e consequentemente pelos teores de RUC delas (Tab. 3). A curva que mais 
se ajustou aos parâmetros foi a linear, com aumento dos minutos de ruminação em função do nível de FDN. Esse comportamento pode ser explicado pelo escopo do próprio trabalho, que visou aumentar os teores de FDN por meio da substituição do concentrado por teores crescentes de resíduo com alto teor de fibra, ou seja, quanto maior o teor de fibra no rúmen, maior a necessidade de ruminação e degradação dela, consequentemente maior tempo despendido para tal.

Tabela 3. Médias, coeficientes de variação (CV, \%), equações de regressão e coeficientes de determinação $\left(\mathrm{R}^{2}\right)$ para consumo e eficiências de ruminação e mastigação, em função dos teores de FDN da dieta

\begin{tabular}{|c|c|c|c|c|c|c|c|c|}
\hline \multirow{2}{*}{ Item } & \multicolumn{5}{|c|}{ Nível de FDN (\%) } & \multicolumn{2}{|c|}{ Genótipo } & \multirow{2}{*}{$\begin{array}{l}\text { CV } \\
(\%)\end{array}$} \\
\hline & 49 & 54 & 59 & 64 & 69 & $\mathrm{~S}$ & BS & \\
\hline \multicolumn{9}{|c|}{ Consumo } \\
\hline MS $(g / d)^{1}$ & 1603,3 & 1649,9 & 1535,8 & 1308,4 & 1023,7 & 1460,72 & 1380,8 & 15,28 \\
\hline $\operatorname{FDN}(g / d)^{2}$ & 697,7 & 857,1 & 883,4 & 873,5 & 773,5 & 846,0 & 787,2 & 15,84 \\
\hline \multicolumn{9}{|c|}{ Eficiência ruminação } \\
\hline $\mathrm{min} / \mathrm{kg} \mathrm{MS}^{3}$ & 133,6 & 162,3 & 210,1 & 258,8 & 378,5 & 217,8 & 239,5 & 37,20 \\
\hline $\mathrm{min} / \mathrm{kg} \mathrm{FDN}^{4}$ & 299,9 & 322,3 & 364,1 & 383,5 & 495,83 & 356,2 & 385,6 & 32,46 \\
\hline \multicolumn{9}{|c|}{ Eficiência mastigação } \\
\hline $\mathrm{min} / \mathrm{kg} \mathrm{MS}{ }^{5}$ & 380,1 & 439,0 & 470,0 & 576,5 & 831,7 & 496,9 & 582,0 & 32,51 \\
\hline $\mathrm{min} / \mathrm{kgFDN}$ & 868,1 & 846,6 & 817,7 & 858,9 & 1087,3 & $832,5 a$ & $958,8 b$ & 24,12 \\
\hline
\end{tabular}

$\mathrm{S}=$ Saanen; BS= 1/2 Boer x Saanen; *Letras minúsculas entre colunas diferem entre si; CV em \%; ${ }^{1} \hat{\mathrm{y}}=$ $4412.8+231.6 X-2.2 X^{2}\left(r^{2} 0.99\right) ;{ }^{2} \hat{y}=-4792.4+189.4 X-1.5 X^{2}\left(r^{2} 0.96\right) ;{ }^{3} \hat{y}=-463,23+11,73 X\left(r^{2} 0.92\right),{ }^{4} \hat{y}=-$ $176,49+9,2787 X\left(r^{2} 0.88\right) ;{ }^{5} \hat{y}=-3894.2-136.7 X+1.3 X^{2}\left(r^{2} 0.976\right) ; \hat{y}=-5949,13-183,07 X+1.6 X^{2}\left(r^{2} 0.91\right)$; em que $\hat{y}=$ tempo gasto com cada atividade alimentar e $\mathrm{X}=$ teores de FDN.

As eficiências de mastigação foram os reflexos das eficiências de ruminação, portanto apresentaram maior tempo despendido com mastigação por $\mathrm{kg}$ de alimento ingerido, à medida que se aumentou o nível de FDN e RUC na dieta. Apesar disso, a curva que melhor se ajustou às eficiências de mastigação foi a quadrática, a curva linear apresentou-se significativa, mas com baixo coeficiente de determinação, portanto baixa adequação dos valores à curva, devido ao fato de o aumento no tempo de mastigação ter ocorrido de forma similar até a dieta com $59 \%$ de FDN e ter aumentado em grandezas muito maiores nas dietas subsequentes com maiores concentrações de FDN.

Vale ressaltar que esse comportamento pode ter sido causado por uma suposta deficiência de proteína disponível para as bactérias ruminais nas dietas com porcentagem de FDN além de $59 \%$ e grandes quantidades de RUC. Isso porque a proteína do resíduo se caracteriza por apresentar resistência à degradação ruminal; segundo Clark et al. (1987), 50\% da proteína é não degradada no rúmen (PNDR), podendo variar em função dos cereais utilizados na fabricação da cerveja. Como o feno utilizado possuía baixa quantidade de $\mathrm{PB}$, as dietas com pouco concentrado e muito RUC podem ter sido deficientes, o que pode ter provocado deficiência proteica, ocasionando redução no crescimento de bactérias ruminais e aumento do tempo de degradação e do tempo de permanência do material fibroso no rúmen, estimulando maior tempo de ruminação com maior salivação e reciclagem de ureia endógena para compensar a deficiência de PNDR no rúmen.

Verificou-se que a utilização de RUC na dieta, como alternativa para aumentar a efetividade da fibra, pode ser feita, pois o resíduo estimula a mastigação, característica importante para manutenção do nível ideal de tampão no rúmen, evitando distúrbios metabólicos; no entanto, altas quantidades devem ser usadas com ressalva, já que suas características nutricionais podem prejudicar a disponibilidade de energia e proteína para o animal. 


\section{CONCLUSÕES}

Animais alimentados com dietas com altos teores de FDN em função do acréscimo de resíduo úmido de cervejaria despendem mais tempo com ruminação, podendo o resíduo ser utilizado como fonte de fibra efetiva. Os genótipos não apresentam diferença quanto ao comportamento alimentar de animais alimentados com RUC.

\section{REFERÊNCIAS BIBLIOGRÁFICAS}

BROCHIER, M.A.; CARVALHO, S. Efeito de diferentes proporções de resíduo úmido de cervejaria sobre as características da carcaça de cordeiros terminados em confinamento. Arq. Bras. Med. Vet. Zootec., v.61, p.190-195, 2009.

BÜRGER, P.J.; PEREIRA, J.C.; QUEIROZ, A.C. Comportamento ingestivo em bezerros Holandeses alimentados com dietas contendo diferentes teores de concentrado. Rev. Bras. Zootec., v.29, p.236-242, 2000.

CABRAL FILHO, S.L.S.C. Avaliação do resíduo de cervejaria em dietas de ruminantes através de técnicas nucleares e correlatas. 1999. 82f. Dissertação (Mestrado) - Centro de Energia Nuclear na Agricultura, Universidade de São Paulo, Piracicaba, SP.

CARVALHO, G.G.P.; PIRES, A.J.V.; SILVA, F.F. et al. Comportamento ingestivo de cabras leiteiras alimentadas com farelo de cacau ou torta de dendê. Pesq. Agropec. Bras., v.39, p.919-925, 2004.

CARVALHO, S.; RODRIGUES, M.T.; BRANCO, R.H. et al. Comportamento ingestivo de cabras alpinas em lactação alimentadas com dietas contendo diferentes níveis de fibra em detergente neutro proveniente de forragem. Rev. Bras. Zootec., v.35, p.562-568, 2006.

CLARK, J.H.; MURPHY, M.R.; CROOKER, B.A. Supplying the protein need soft dairy cattle from by products feeds. J. Dairy Sci.,v.70, p.1092-1109, 1987.

COSTA, C.O.; FISCHER, V.; VETROMILLA, M.A.M. Comportamento ingestivo de vacas Jersey confinadas durante a fase inicial da lactação. Rev. Bras. Zootec.,v.32, p.418-424, 2003.
DADO, R.G.; ALLEN, M.S. Intake limitation, feeding behavior and rumen function of cows challenged with rumen fill from dietary fiber or inert bulk. J. Dairy Sci., v.78, p.118-133, 1995.

DESWYSEN, A.G.; DITILLEUL, P.; GODFRIN, J.P. et al. Nycterohemeral eating and ruminanting patterns in heifers fed grass or corn silage: analysis by finite fourier transform. $J$. Anim. Sci., v.71, p.2739-2747, 1993.

FERREIRA, D.F. Sisvar: Sistema de análise de variância para dados balanceados. Versão 4.0. Lavras: DEX/UFLA, 2000.

FISCHER, V.; DEWYSEN, A.G.; DÈSPRES, L. et al. Padrões nictemerais do comportamento ingestivo de ovinos. Rev. Bras. Zootec., v.27, p.362-369, 1998.

GERON, L.J.V.; ZEOULA, L.M.; ERKEL, J.A. et al. Coeficiente de digestibilidade e características ruminais de bovinos alimentados com rações contendo resíduo de cervejaria fermentado. Rev. Bras. Zootec., v.37, p.16851695, 2008.

MERTENS, D.R. Creating a system for meeting the fiber requirements of dairy cows. J. Dairy Sci., v.80, p.1463-1481, 1997.

NUTRIENT requirements dairy cattle. 7.ed. Washington, DC: National Academy of Sciences, 2001. 408p.

OFFICIAL methods of analysis. Washington, DC: AOAC, 1984, 1141p.

RIBEIRO, V.L.; BATISTA, A.M.V.; CARVALHO, F.F.R. et al. Comportamento ingestivo de caprinos Moxotó e Canindé submetidos a alimentação à vontade e restrita. Acta Sci. Anim. Sci., v.28, p.331-337, 2006.

SISTEMA de análises estatísticas. Versão 8.0. Viçosa: UFV, 1999. 150p.

VAN SOEST, P.J.; ROBERTSON, J.B.; LEWIS, B.A. Symposium: carbohydrate methodology, metabolism, and nutritional implications in dairy cattle. J. Dairy Sci., v.74, p.3583-3597, 1991.

WEST, J.W.; MARTIN, L.O.E.S. Wet brewers grains for lactating dairy cows during hot, humid weather. J. Dairy Sci., v.77, p.196-204, 1994. 